\title{
¿Pressure Fields in the Airflow over Wind-Generated Surface Waves
}

\author{
Christoph S. Funke, ${ }^{\mathrm{a}, \mathrm{b}}$ MARC P. Buckley, ${ }^{\mathrm{a}}$ Larissa K. P. Schultze, ${ }^{\mathrm{a}}$ Fabrice Veron, ${ }^{\mathrm{c}}$ \\ MARY-LOUISE E. TIMMERMANS, ${ }^{b}$ AND JEFFREY R. CARPENTER ${ }^{a}$ \\ ${ }^{a}$ Institute of Coastal Ocean Dynamics, Helmholtz-Zentrum Hereon, Geesthacht, Germany \\ ${ }^{\mathrm{b}}$ Department of Earth and Planetary Sciences, Yale University, New Haven, Connecticut \\ ${ }^{\mathrm{c} S}$ School of Marine Science and Policy, University of Delaware, Newark, Delaware
}

(Manuscript received 17 December 2020, in final form 3 September 2021)

\begin{abstract}
The quantification of pressure fields in the airflow over water waves is fundamental for understanding the coupling of the atmosphere and the ocean. The relationship between the pressure field and the water surface slope and velocity are crucial in setting the fluxes of momentum and energy. However, quantifying these fluxes is hampered by difficulties in measuring pressure fields at the wavy air-water interface. Here we utilize results from laboratory experiments of wind-driven surface waves. The data consist of particle image velocimetry of the airflow combined with laser-induced fluorescence of the water surface. These data were then used to develop a pressure field reconstruction technique based on solving a pressure Poisson equation in the airflow above water waves. The results allow for independent quantification of both the viscous stress and pressure-induced form drag components of the momentum flux. Comparison of these with an independent bulk estimate of the total momentum flux (based on law-of-the-wall theory) shows that the momentum budget is closed to within approximately $5 \%$. In the partitioning of the momentum flux between viscous and pressure drag components, we find a greater influence of form drag at high wind speeds and wave slopes. An analysis of the various approximations and assumptions made in the pressure reconstruction, along with the corresponding sources of error, is also presented.
\end{abstract}

KEYWORDS: Atmosphere-ocean interaction; Wind waves; Measurements; Gravity waves

\section{Introduction}

The vast majority of the mechanical energy exchange between the atmosphere and ocean occurs through the ocean surface wave field (Wunsch and Ferrari 2004). Winds blowing over the surface of the ocean generate surface gravity waves, and a host of processes arising from the wavy surface then modify the exchanges of momentum, energy, and gases in the near-surface boundary layer between ocean and atmosphere. The alterations in these fluxes due to surface waves are known to have important implications for the climate system (Cavaleri et al. 2012), as well as in weather prediction (Zhang et al. 2006) and ocean circulation (Ardhuin et al. 2004).

A principal barrier to understanding and quantifying the exchange of momentum and energy at the air-water interface is the extreme difficulty in performing measurements near a moving wavy interface. To quantify atmosphereocean fluxes it is necessary to measure velocities, pressure, and interface displacements simultaneously, at distances very close to the moving air-water interface, and over scales small enough to resolve wave phases. While a number of coupled physical processes influence the air-water momentum budget (e.g., wave breaking, airflow separation),

\footnotetext{
D Denotes content that is immediately available upon publication as open access.
}

Corresponding author: J. R. Carpenter, jeff.carpenter@ hereon.de momentum is directly exchanged between the air and the water either through surface viscous stresses or pressureinduced form drag (e.g., Phillips 1977). The relative importance of each of these viscous and form drag components has been of interest for the past decades. Quantifying their respective roles in the generation of ocean surface currents and waves still remains a major challenge (Sullivan and McWilliams 2010; Grare et al. 2013; Buckley et al. 2020).

Pressure measurements within the air-side boundary layer over wind waves are sparse (e.g., Elliott 1972; Dobson 1971; Hasselmann and Bösenberg 1991; Donelan et al. 2006), with the most recent ones reported by Savelyev et al. (2011) and Grare et al. (2013) from experiments in wind-wave flumes. Particle image velocimetry (PIV) measurements within the water-side (Banner and Peirson 1998) and air-side (Veron et al. 2007; Buckley et al. 2020) viscous sublayers of wind-generated waves have given reliable estimates of viscous drag for laboratory wind waves. Mean form drag contributions were estimated using the law-of-the-wall, and it was suggested that the relative contribution of viscous drag to the air-sea momentum flux dominates over smooth nascent waves, but decreases with increasing wave slope (Buckley et al. 2020). However, to the best of our knowledge, both quantities have never been measured simultaneously. While significant advances have been recently made using both direct numerical simulations (DNS) and large-eddy simulations (LES) of wind-wave dynamics (see e.g., Husain et al. 2019, with matching airflow PIV measurements), modeling efforts suffer from a number of drawbacks. Some of these limitations include either neglecting or not resolving viscous effects at the surface, incomplete 


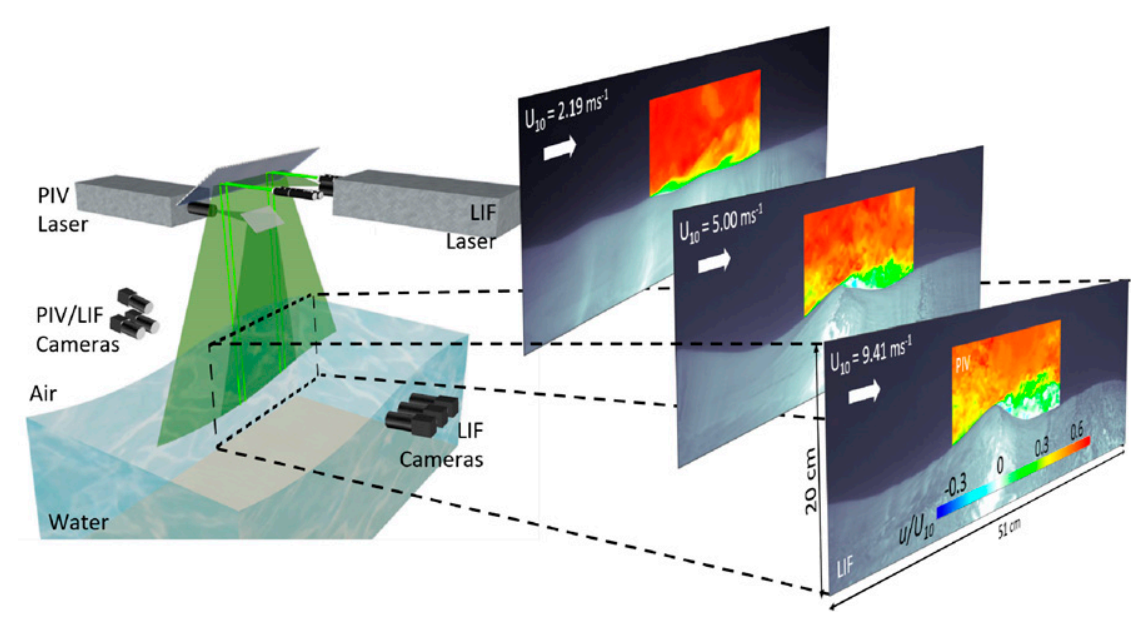

FIG. 1. Sketch of the experimental setup, placed at a fetch (i.e., distance down-wind from the onset of wind-wave growth) of $22.7 \mathrm{~m}$ within University of Delaware's large wind-wave-current facility, along with examples of composite instantaneous two-dimensional LIF-PIV data products, for the three wind speeds examined here $\left(U_{10}=2.2,5.0\right.$, and $\left.9.4 \mathrm{~m} \mathrm{~s}^{-1}\right)$.

air-water coupling physics, and restrictions to unrealistic Reynolds numbers and wave fields (typically monochromatic) (e.g., Sullivan et al. 2000, 2018; Liu et al. 2010; Hao and Shen 2019).

In the present study we use laboratory measurements to simultaneously estimate both the viscous tangential stress and the pressure-induced form stress. This is done by using twodimensional velocity fields measured over wind-generated waves through PIV, together with interface detection from laser-induced fluorescence (LIF). This is necessarily done with an extremely high spatial resolution that is capable of resolving the viscous boundary layer along the air-water interface (Buckley and Veron 2016, 2017). The velocity measurements are then used to estimate an approximate reconstruction of the pressure field based on a Poisson-solver approach (Murai et al. 2007; Van Oudheusden 2013). To our knowledge, the results reported herein are the first spatially resolved pressure fields directly measured above wind-generated waves. The assumptions involved in this method, such as approximate boundary conditions and the limitations of two-dimensional velocity inputs, are systematically tested. We find that the form stress calculated from the reconstructed pressure fields results in a momentum budget that is closed to within $5 \%$. The results allow for a partitioning of momentum fluxes between viscous and form stresses, and demonstrate the increasing importance of form stress at high wind speeds and wave slopes. The accurate reconstruction of pressure fields also opens new possibilities for examination of the physical mechanisms of wind-wave coupling.

The paper is organized as follows. After a brief description of the experimental setup, we describe the methods used in reconstructing the pressure field. Then results of the pressure field reconstruction are presented including a comparison of viscous and form drag stresses for different wind speeds. In the discussion section we test the various assumptions made in our pressure reconstruction method utilizing a large-eddy simulation of turbulent airflow over fixed sinusoidal wave forms. Conclusions are summarized in the final section.

\section{Laboratory experiments}

\section{a. Setup}

The laboratory measurements used in this paper were obtained at University of Delaware's large wind-wave-current facility. Since the experiments are described in detail in Buckley and Veron (2017), we will offer here only a very brief description. A multilaser, multicamera, optical wind-wave measurement system was placed at a fetch of $22.7 \mathrm{~m}$, in the wind-wave-current tank that is $42 \mathrm{~m}$ long, $1 \mathrm{~m}$ wide, and $1.25 \mathrm{~m}$ high. The mean water depth was $0.70 \mathrm{~m}$, with an airflow space of $0.55 \mathrm{~m}$. A sketch of the experimental setup is presented in Fig. 1, along with examples of instantaneous airflow velocity fields obtained by PIV, embedded in larger LIF snapshots of the wave field.

In this paper, measurements from three different wind/wave conditions are used. Winds with mean $10-\mathrm{m}$ equivalent speeds of $U_{10}=2.2,5.0$, and $9.4 \mathrm{~m} \mathrm{~s}^{-1}$ were generated by the recirculating wind tunnel. Wind waves were observed for all three wind speeds and all waves studied here were windgenerated (no paddle generated waves). The different experimental conditions are summarized in Table 1.

Despite the limited domain of the laboratory channel, the mean airflow characteristics within the first $\sim 10-20 \mathrm{~cm}$ above the surface, as well as the statistics of turbulent momentum flux, are in good agreement with past in situ observations and LES simulations of steady state conditions (see Fig. 11 of Buckley and Veron 2016).

\section{b. Data processing and phase averages}

As mentioned above, the waves generated in these laboratory experiments are generated by the wind and are therefore 
TABLE 1. Summary of experimental conditions. Each experiment is characterized by a friction velocity $u *$, and the $10-\mathrm{m}$ extrapolated velocity $U_{10}$, computed by fitting the logarithmic part of the averaged PIV velocity profile in the air. Peak wave frequencies $f_{p}$ were obtained from laser wave gauge frequency spectra. The phase speed $c_{p}$ and wavenumber $k_{p}$ were derived by applying linear wave theory to $f_{p}$. The wave amplitude $a_{p}$ was obtained from rootmean-square amplitude $a_{p}=\sqrt{2} a_{\mathrm{rms}}$ computed from the wave gauge derived water surface elevation time series.

\begin{tabular}{cccccccc}
\hline \hline $\begin{array}{c}U_{10} \\
\left(\mathrm{~m} \mathrm{~s}^{-1}\right)\end{array}$ & $\begin{array}{c}u_{*} \\
\left(\mathrm{~cm} \mathrm{~s}^{-1}\right)\end{array}$ & $\begin{array}{c}c_{p} \\
\left(\mathrm{~m} \mathrm{~s}^{-1}\right)\end{array}$ & $\begin{array}{c}c_{p} / u_{*} \\
(-)\end{array}$ & $\begin{array}{c}a_{p} \\
(\mathrm{~cm})\end{array}$ & $\begin{array}{c}\lambda_{p} \\
(\mathrm{~m})\end{array}$ & $\begin{array}{c}a_{p} k_{p} \\
(-)\end{array}$ & $\begin{array}{c}f_{p} \\
(\mathrm{~Hz})\end{array}$ \\
\hline 2.2 & 7.3 & 0.47 & 6.5 & 0.15 & 0.14 & 0.07 & 3.3 \\
5.0 & 17 & 0.62 & 3.7 & 0.5 & 0.25 & 0.13 & 2.5 \\
9.4 & 31 & 0.78 & 2.5 & 1.20 & 0.39 & 0.19 & 2.0 \\
\hline
\end{tabular}

not monochromatic waves. However, at short laboratory fetches, the wave field is relatively narrow banded. Furthermore, it is generally useful to examine the data and their variations relative to the phase of an equivalent idealized periodic water wave. To do so, we average our data relative to the local wave phase and height above the water surface (see Fig. 2). Throughout the rest of the paper, we refer to such an average as the phase average. In this section, we describe our method of phase averaging the data.

The details of the wave phase averaging procedure are as follows: first and foremost, phase averaging requires a method of reliably determining wave phase along each part of the water surface. This is done using the Hilbert transform of the water surface profile, identical to the phase detection in Buckley and Veron (2017). Figure 2 shows a typical wave profile in a PIV image. We begin the phase averaging process by defining a new coordinate system $(\phi, \zeta)$ in the region above the wave,

$$
\phi(x, z)=\phi(x) \quad \text { and } \quad \zeta(x, z)=z-\eta(x) .
$$

In this coordinate system, the $\phi$ coordinate represents the wave phase, and $\eta$ the water surface elevation. A wave phase of 0 denotes the wave crest and a wave phase of $\pm \pi$ denotes the wave trough (see abscissa in Fig. 2). The new vertical coordinate $\zeta$ measures distance above the water surface.

To obtain phase-averaged fields, we bin data into 144 uniformly sized phase bins covering the interval $-\pi<\phi \leq \pi$. We average data from all PIV snapshots that are within the same $\phi$ and $\zeta$ bin. The resultant phase average has the same vertical resolution as the original data but only 144 grid points in the horizontal direction (one per phase bin). The phase average of an arbitrary field $\Xi$ (which can be a PIV velocity field or any other derived product such as the vorticity) is denoted by $\langle\Xi\rangle$.

\section{Pressure reconstruction method}

For incompressible flows in fluids of homogeneous density, the pressure is related to gradients in velocity through the following pressure Poisson equation

$$
-\nabla^{2} p=f \quad \text { with } \quad f \equiv \rho \nabla \cdot(\mathbf{u} \cdot \nabla) \mathbf{u} .
$$

Here, $p$ is the deviation of pressure from its mean hydrostatic level; $\rho$ is the air density; and $\mathbf{u}=(u, v, w)$ is the velocity vector

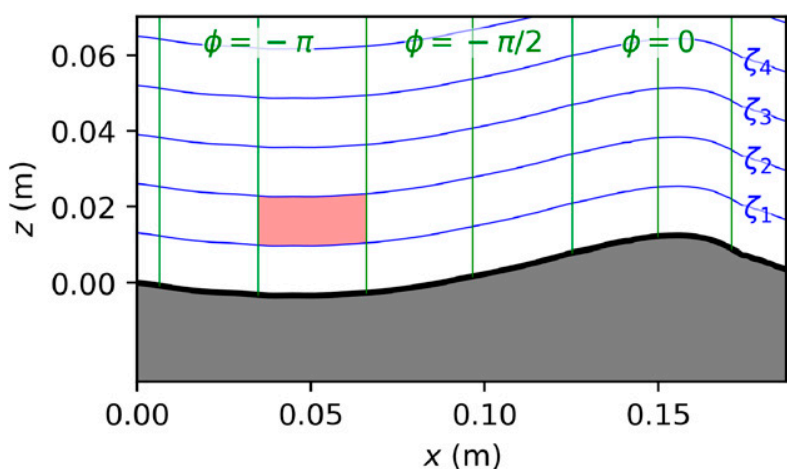

FIG. 2. Plot of the coordinate system used in the analysis. The green lines indicate a constant phase $(\phi)$, as detected using the Hilbert transform method. The blue lines indicate lines of constant $\zeta$ and the black line is the water surface $(\zeta=0)$. The red region exemplifies a bin as used in the phase averaging process, albeit much bigger than those actually used. All values within the same bin are averaged together.

in the standard Cartesian $(x, y, z)$ coordinate system. We use the $\equiv$ symbol to denote definitions. Equation (2) is derived by taking the divergence of the Navier-Stokes equations. It has been shown to yield reliable pressure fields from PIV data in engineering applications (Murai et al. 2007; Van Oudheusden 2013).

The quantity $f$ will be referred to as the forcing function of the system. It is proportional to the second-order invariant of the velocity gradient tensor, and because it is independent of the frame of reference, it can be meaningfully interpreted. In fact, $f$ can be split into two different contributions, strain and vorticity (or enstrophy):

$$
f \equiv \rho \nabla \cdot(\mathbf{u} \cdot \nabla) \mathbf{u}=\rho\left(\mathbf{S}: \mathbf{S}-\frac{1}{2} \mathbf{q} \cdot \mathbf{q}\right)
$$

where $\mathbf{S} \equiv(1 / 2)\left[\nabla \mathbf{u}+(\nabla \mathbf{u})^{\mathrm{T}}\right]$ is the strain tensor, and $\mathbf{q} \equiv \nabla \times \mathbf{u}$ the vorticity. This comes from the fact that $\nabla \cdot(\mathbf{u} \cdot \nabla) \mathbf{u}=$ $(\mathbf{u} \cdot \nabla)(\nabla \cdot \mathbf{u})+\nabla \mathbf{u}: \nabla \mathbf{u}$, with the first term vanishing, and the second rewritten as $\mathbf{S}: \mathbf{S}-(1 / 2) \mathbf{q} \cdot \mathbf{q}$ (see Davidson 2015, p. 45). The strain component is positive since $\mathbf{S}: \mathbf{S}=\alpha^{2}+\beta^{2}+\gamma^{2}>0$, with $\alpha, \beta, \gamma$ the principal rates of strain. Also, the enstrophy component is always negative, i.e., $-(1 / 2) \mathbf{q} \cdot \mathbf{q}<0$. Therefore, $f$ gives a local measure of the relative importance of strain and vorticity. If $f<0$, the flow is dominated by vorticity (i.e., has high enstrophy), whereas if $f>0$ it is dominated by strain. Equivalently, regions of high vorticity (strain) lead to $\nabla^{2} p>0$ $\left(\nabla^{2} p<0\right)$ such that there is forcing toward a local minimum (maximum) in the pressure field.

Due to the limitations of the PIV data in measuring only two-dimensional, $x-z$ planar flows, it is not possible to use the full $f$ to force the pressure Poisson equation. We will therefore proceed by tentatively assuming that the deviations from planarity cancel out over long time averages. This assumption is discussed in section 5, in which turbulence-resolving numerical simulations are used to demonstrate that accurate average pressure fields and form drag estimates are obtained. Utilizing only the planar terms, the forcing function simplifies to 


$$
f=-2 \rho\left(\frac{\partial u}{\partial x} \frac{\partial w}{\partial z}-\frac{\partial u}{\partial z} \frac{\partial w}{\partial x}\right) .
$$

Alternate forms of $f$ are possible with the same assumptions on the airflow (see, e.g., Van Oudheusden 2013). These formulations are identical for perfectly two-dimensional flows, but may lead to slightly different results when deviations from planarity exist. We have chosen the above formulation because it gives an unbiased estimate of $f$ when there is random noise in the velocity gradient data. Once $f$ is determined from the PIV measurements, the pressure field is readily estimated.

\section{a. Numerical procedure}

To solve the pressure Poisson equation (2), we use a finite difference scheme. On the interior of the domain, at coordinate indices $(i, j)$,

$$
\frac{p_{i+1, j}-2 p_{i, j}+p_{i-1, j}}{(\Delta x)^{2}}+\frac{p_{i, j+1}-2 p_{i, j}+p_{i, j-1}}{(\Delta z)^{2}}=-f_{i, j},
$$

where $\Delta x$ and $\Delta z$ are the grid spacings in the horizontal $(x)$ and vertical $(z)$ directions. The finite differences algorithm is implemented on a square grid (with $\Delta x=\Delta z$ ) coinciding with the experimental measurements.

The computational domain is rectangular except that the bottom boundary is taken as the wavy water surface, and is identical to the PIV measurement domain. Boundary conditions on the bottom water surface are implemented using a combination of the numerical methods described by Fox (1944), Noye and Arnold (1990), and Morton and Mayers (2005). Wave-height measurements, described previously in Buckley and Veron (2016), are used to determine the position and slope of the bottom boundary over subgrid scales. The key results presented in this paper do not change significantly when simpler boundary approximations involving the discretization of the bottom water surface are used [see Noye and Arnold (1990) for a description of the standard method]. Thus, the results presented here are independent of the interpolation scheme used at the bottom boundary. The side and top boundaries are located at the edges of the PIV field.

\section{b. Boundary conditions}

For each PIV field, instantaneous pressure fields are computed. At the air-water interface, we use the Neumann condition where

$$
\left.\nabla p\right|_{0} \cdot \mathbf{n}=-\left.\rho \frac{D \mathbf{u}}{D t}\right|_{0} \cdot \mathbf{n}+\left.\rho \nu \nabla^{2} \mathbf{u}\right|_{0} \cdot \mathbf{n},
$$

with $\nu$ the kinematic viscosity, along the air side of the water surface, denoted by the subscript 0 . This condition is derived by taking the dot product between the unit vector normal to the water surface, $\mathbf{n}$, pointing into the air, and the Navier-Stokes equations by evaluating the equations in the limit as the airwater interface is approached from above.

The acceleration of the water surface [first term on the right-hand side of Eq. (6)] is computed by decomposing the surface measurements into spatial Fourier components. We assume that each individual wave component propagates with an angular frequency given by the deep water gravitycapillary wave dispersion relationship

$$
\omega=\sqrt{k\left(g+\frac{\sigma k^{2}}{\rho_{w}}\right)} .
$$

Here, $k$ is the wavenumber; $g$ is the acceleration due to gravity; $\rho_{w}$ is the water density; and $\sigma=0.074 \mathrm{~N} \mathrm{~m}^{-1}$ is the surface tension. Note that alterations in this dispersion relation due to interaction with wind are small, of the order of the ratio of the air to water densities, and are therefore neglected (e.g., Young and Wolfe 2014). The acceleration of the water surface is then computed by calculating and summing accelerations of individual wave components using linear wave theory. At every step throughout the calculation, the surface profiles (displacement, velocity, and acceleration) are smoothed using a second-order low-pass Butterworth filter with a cutoff wavelength of $1 \mathrm{~cm}$.

To estimate the spatial velocity gradients in the viscous term of Eq. (6), the instantaneous velocities measured by PIV were first fitted with cubic smooth spline shells, and gradients were subsequently estimated using analytical derivatives of the spline fits [for additional details, see Buckley and Veron (2017), and references therein]. Since the wind-wave conditions are within the transitionally rough regime in all three experiments (with Reynolds roughness numbers greater than 0.2 , see for example the classification proposed by Kitaigorodskii and Donelan 1984; Donelan 1998), the near-surface instantaneous velocities fluctuate significantly in the vertical and streamwise directions, and higher-order spatial derivatives are expected to show significant variability. However, the spatial resolution of the measurements used here is such that the viscous sublayer is fully resolved in all wind speed conditions. A sensitivity analysis is performed in section 5 to examine any errors arising in the treatment of the viscous term [second term on the right-hand side of Eq. (6)].

On the side boundaries, we use the Neumann condition $\nabla p$. $\mathbf{n}=0$. This boundary condition may lead to a distortion of the pressure field near the side of the computational domain. As a result, when doing subsequent pressure calculations, we only include pressure values on the interior $60 \%$ of the domain. The improvement in doing so is discussed in section 5 .

On the top boundary, we use the boundary condition $p=0$. This Dirichlet condition ensures that the Poisson problem is well posed. Pressure perturbations caused by the waves decay away from the water surface, making the approximation a good one for small amplitude waves, and relatively large domain sizes.

\section{Results}

\section{a. Instantaneous fields}

Figure 3 shows $u, w, f$, and $p$ for a single PIV field in the $U_{10}=5.0 \mathrm{~m} \mathrm{~s}^{-1}$ wind speed experiment. The figure highlights the different steps used to compute pressure fields. First, the PIV data yields $u$ and $w$ velocity fields. As suggested from the $u$ field, and confirmed by an analysis of the vorticity field (not shown; see Buckley et al. 2020), airflow separation occurs 

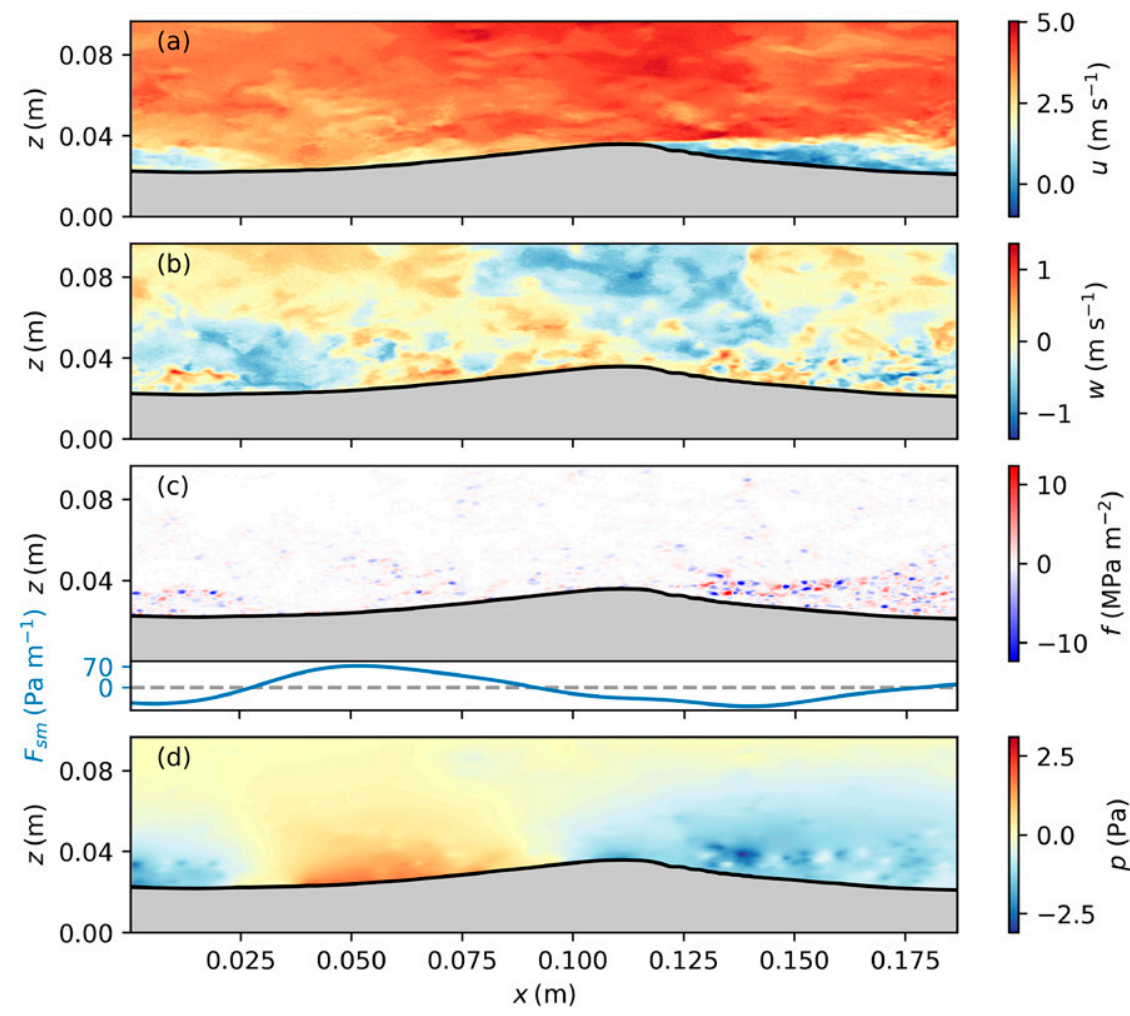

FIG. 3. Plot of (a) horizontal velocity $u$, (b) vertical velocity $w$, (c) pressure forcing $f$, and (d) pressure $p$, for one sample PIV field of the $U_{10}=5.0 \mathrm{~m} \mathrm{~s}^{-1}$ wind speed case. The upper panel of (c) shows the instantaneous $f$, while the lower panel shows $F_{\mathrm{sm}}$, obtained from $f$ vertically integrated over the first $3 \mathrm{~cm}$ above the water surface, then smoothed using a Gaussian filter with $3.5-\mathrm{cm}$ half power width. The wind is flowing from the left and the wave is propagating rightwards. The forcing function $f$ was computed from the $u$ and $w$ fields using (4). The instantaneous pressure field was then calculated from $f$ using the method described in section 3 .

starting at the wave crest and extends down the entire leeward face of the wave (Fig. 3a). Figure 3b shows that variability in the vertical velocity, $w$, is strongest at the boundary between the free stream and the separated flow on the leeward side of the wave crest. The high variability in $w$ indicates that turbulence is strongest in this region, which is characterized by a separated shear region (Buckley and Veron 2016). Slight trends of upward (downward) air motion exist on the windward (leeward) side of the wave. In general, wave-coherent velocities of the surface waves are difficult to distinguish in the instantaneous velocity fields of the airflow, but become apparent once suitable averaging is performed. For time average wavecoherent motions, see Buckley and Veron (2019).

Next, we estimate the forcing function $f$ from the PIV velocity fields using (4). As can be seen, $f$ has large variability over the entire PIV field (Fig. 3c, top). Since $f$ is computed from spatial gradients in $(u, w)$, it highlights the small-scale turbulent variability that is present. This variability is strongest on the leeward side of the wave, reflecting the fact that the flow is most turbulent in this region. The bottom panel of Fig. 3c shows $f$ vertically integrated over the first $3 \mathrm{~cm}$ above the water surface, then smoothed using a $3.5 \mathrm{~cm}$ width-at-halfmaximum Gaussian filter. This curve will be denoted as $F_{\mathrm{sm}}$ and gives an estimate of the net $f$ forcing close to the water surface, without the turbulent variability. As can be seen, $F_{\mathrm{sm}}$ is positive on the windward side of the wave where the surface shear is maximum. In contrast, $F_{\mathrm{sm}}$ tends to be negative in the separated region on the leeward side of the wave, indicating that this is a region of high vorticity. Despite the high $f$ variability present, the smallscale regions of strong turbulent strain and vorticity largely cancel one another in the separated lee of the wave, and the smoothed near-surface distribution of $F_{\text {sm }}$ exhibits comparable amplitudes on the windward and leeward wave faces.

Finally, from the forcing function we estimate the instantaneous pressure field using the procedure described above. The pressure field (Fig. 3d) obtained from the Poisson solver resembles the smoothed, integrated version of the forcing function. High pressure is located on the strain-dominated windward face of the wave. On the other hand, low pressure is located on the vorticity-dominated leeward side of the wave crest. The overall pressure response is comparable in magnitude on both the windward and leeward sides. The large turbulent variability in $f$ is responsible for creating localized eddy structures, most clearly visible on the leeward side of the wave (Fig. 3d).

The instantaneous pressure field shown here is primarily intended to demonstrate the method of computing pressure 
fields. Due to nonplanar turbulent structures in the flow (i.e., with an out-of-plane $y$ component), the instantaneous fields obtained in the Poisson solver are expected to depart substantially from actual instantaneous pressure fields. We show in section 5, however, that once the instantaneous fields are suitably phase-averaged, the resulting form drag shows relatively small error, indicating that the pressure field is also a relatively unbiased estimate. For the remainder of the paper, we therefore focus only on phase-averaged quantities.

\section{b. Phase-averaged pressure fields}

The pressure fields obtained by phase averaging instantaneous pressure fields (such as those above) are shown in Fig. 4, with each panel corresponding to a different wind speed case.

For the $U_{10}=2.2 \mathrm{~m} \mathrm{~s}^{-1}$ wind speed case, the pressure field is approximately $90^{\circ}$ out of phase with the water surface displacement. This configuration of the pressure field, with high pressure on the windward side and low pressure on the leeward side of the wave crest, is optimal for transferring momentum to the water (see below).

For the two higher wind speed cases, the pressure field shifts upwind and becomes nearly in phase with the water wave elevation. Nonetheless, a small phase shift is present, with maximum and minimum pressures located slightly leeward of the wave trough and crest, respectively. Note that the amplitude of the pressure field variations increases by roughly one order of magnitude with each successive wind speed case.

\section{c. Momentum flux}

The average flux of momentum across the water surface is determined by the sum of both a viscous stress acting tangential to the water surface, and a pressure-induced form drag acting normal to it. The horizontal component of these two stresses can be calculated as

$$
\bar{\tau}_{v}=\rho \nu \overline{\left.\left(\frac{\partial u}{\partial z}+\frac{\partial w}{\partial x}-2 \frac{\partial u}{\partial x} \frac{\partial \eta}{\partial x}\right)\right|_{0}}
$$

and

$$
\bar{\tau}_{f}=\overline{\left.p\right|_{0} \frac{\partial \eta}{\partial x}}
$$

for the viscous and form drags, respectively. In these equations the overbar refers to an ensemble average for all measurements in an experiment. These equations are derived from the stress tensor for incompressible Newtonian fluids (see appendix A for details). Due to difficulties in measuring velocity gradients directly at the water surface, and to reduce measurement noise, we use all PIV measurements within the viscous sublayer when calculating the viscous stress. The viscous sublayer thickness is defined here as five wall units, $\nu / u_{*}$, from the water surface. Here, $u *$ refers to the friction velocity.

In Fig. 4, we also plot the pressure-slope correlations, $\left\langle\left. p\right|_{0} \partial \eta / \partial x\right\rangle$, below each phase-averaged pressure field. Here again, the subscript $\left.\right|_{0}$ denotes the surface values. To a good approximation, the average value of this curve yields the value of $\bar{\tau}_{f}$, quantifying the mean pressure-induced flux of horizontal momentum across the water surface. The exact calculation of $\bar{\tau}_{f}$ requires the average to be weighted slightly in order to accommodate a
TABLE 2. Table of bulk momentum budget components over water waves for each wind speed. Mean viscous stress $\bar{\tau}_{v}$, form drag $\bar{\tau}_{f}$, and total stress $\tau_{\text {tot }}^{*}$ were calculated using Eqs. (8), (9), and (11).

\begin{tabular}{ccccc}
\hline \hline$U_{10}\left(\mathrm{~m} \mathrm{~s}^{-1}\right)$ & $u *\left(\mathrm{~cm} \mathrm{~s}^{-1}\right)$ & $\bar{\tau}_{v} / \tau_{\text {tot }}^{*}$ & $\bar{\tau}_{f} / \tau_{\text {tot }}^{*}$ & $\bar{\tau}_{\text {tot }} / \tau_{\text {tot }}^{*}$ \\
\hline 2.2 & 7.3 & 0.86 & 0.15 & 1.01 \\
5.0 & 17 & 0.60 & 0.39 & 0.99 \\
9.4 & 31 & 0.40 & 0.65 & 1.05 \\
\hline
\end{tabular}

nonuniform phase distribution of data points, due to deviations of the observed waveforms from perfect sinusoids. The $\bar{\tau}_{f}$ values estimated in this way are listed in Table 2.

For the $U_{10}=2.2 \mathrm{~m} \mathrm{~s}^{-1}$ experiment, the optimal $90^{\circ}$ phase shift between the pressure field and the surface elevation indeed leads to a pressure-slope correlation that is positive at nearly every phase. The form drag curve has two maxima along the wave profile. The first maximum occurs windward of the wave trough and the second maximum is just leeward of the wave crest. These are the regions where the pressure field most strongly influences wave growth since the interface slope is a maximum. Most of the transfer of horizontal momentum at the air-sea interface is split evenly between the windward and leeward sides of the wave, as evidenced by the fact that the pressureslope correlation curves are of a similar magnitude there.

For the two higher wind speed cases, the phase shift between the pressure field and the surface elevation is reduced (less than $90^{\circ}$ ). Yet, the pressure-slope correlation exhibits a skew toward positive values indicating an overall momentum transfer which leads to a positive form drag.

Summing together (8) and (9) we estimate the average total stress on the water surface (i.e., the total flux of horizontal momentum across the water surface) by

$$
\bar{\tau}_{\text {tot }}=\bar{\tau}_{f}+\bar{\tau}_{v} .
$$

To validate the results, we also compute an independent estimate of the total stress using law-of-the-wall theory as

$$
\tau_{\mathrm{tot}}^{*}=\rho u_{*}^{2}
$$

The friction velocity $u_{*}$ can be estimated from $d U / d z$, the mean flow shear within the logarithmic layer. Here $U(z)$ denotes the ensemble mean of the air velocity at height $z$. Values of $u_{*}$ for each wind speed were first reported and described by Buckley and Veron (2016). In this analysis, we use these reported values.

Table 2 shows the estimates of $\bar{\tau}_{\text {tot }}, \bar{\tau}_{v}$, and $\bar{\tau}_{f}$ for each of the wind speeds as calculated with Eqs. (8)-(10). For all three wind speed cases, our estimated viscous and form stresses close the momentum budget to within $5 \%$ of total stress. As wind speed increases, the contribution of form drag to the total momentum flux increases. For the $U_{10}=2.2 \mathrm{~m} \mathrm{~s}^{-1}$ wind speed, form contributes only $15 \%$ to the total horizontal momentum flux across the surface. However, at the largest wind speed of $U_{10}=$ $9.4 \mathrm{~m} \mathrm{~s}^{-1}$, form drag is the dominant component contributing $65 \%$ of the momentum flux. Note that we cannot separate the effects of wind speed (or wave age) variations from those of 

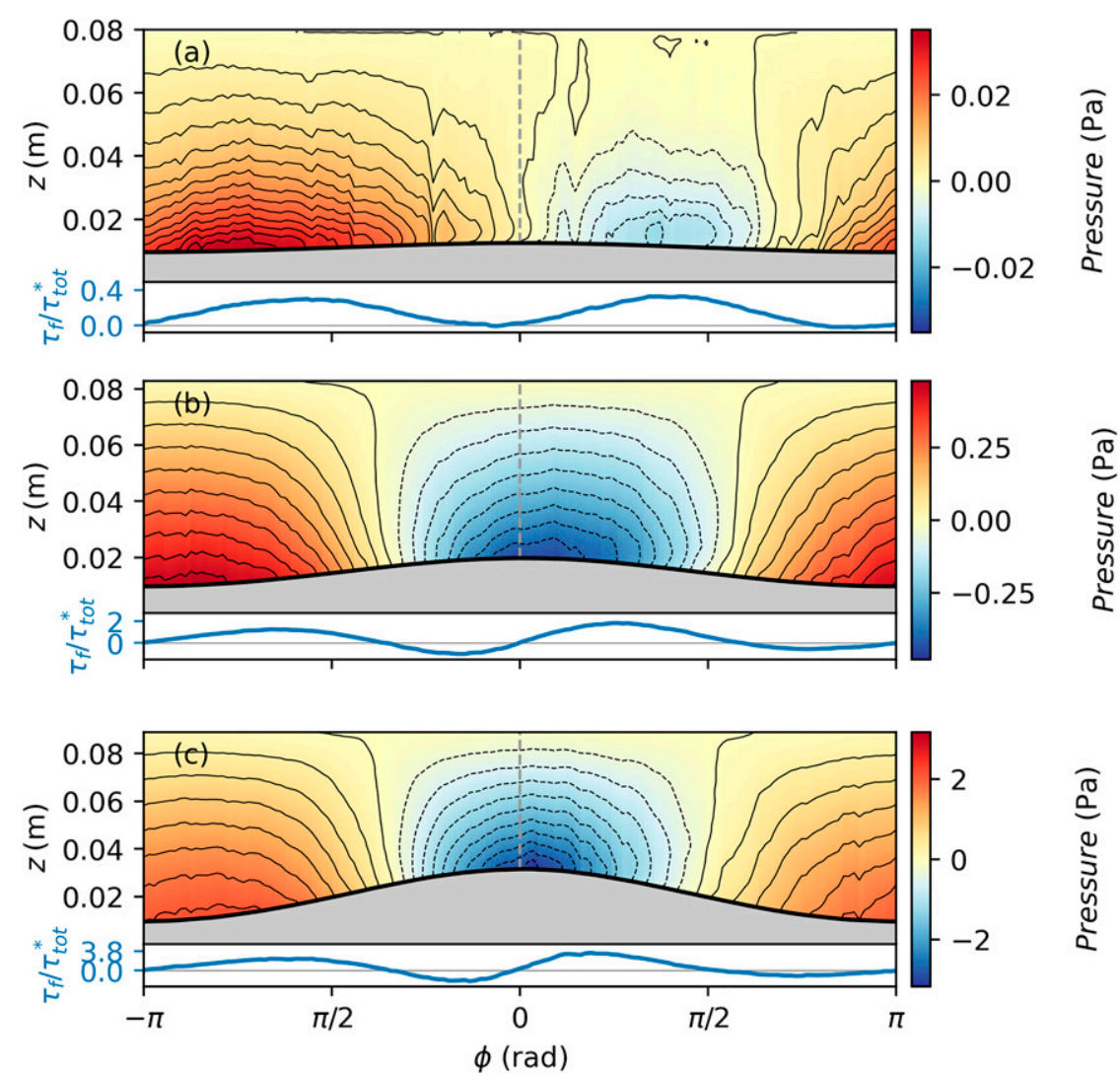

FIG. 4. Phase average pressure fields over wind-driven water waves for wind speeds (a) $U_{10}=2.2 \mathrm{~m} \mathrm{~s}^{-1}$, (b) $U_{10}=5.0 \mathrm{~m} \mathrm{~s}^{-1}$, and (c) $U_{10}=9.4 \mathrm{~m} \mathrm{~s}^{-1}$. The wind is flowing from the left and the wave is propagating rightwards. The dashed gray lines indicate the location of the wave crest $(\phi=0)$. Below each pressure field is a plot showing the pressure-slope correlation averaged by wave phase. Values of pressure slope correlation are normalized by total stress, as estimated using (11).

wave slope variations since these are tightly related in these experiments at fixed fetch.

In Fig. 5, we show our mean normalized form drag estimates $\bar{\tau}_{f} / \tau_{\text {tot }}^{*}$, as a function of wave slope, alongside estimates from previous laboratory pressure measurements (Grare 2009; Savelyev 2009), LES (Hara and Sullivan 2015), and DNS (Sullivan et al. 2000; Yang and Shen 2010). Since wave age is also suspected of influencing form drag (e.g., Sullivan et al. 2000), only relatively young wave age conditions were selected here $\left(c_{p} / u_{*}<8\right)$, including a stationary wave case from Sullivan et al. (2000). We observe a good agreement in our form drag estimates with previous studies, in spite of differences in fetch, wave age, and the mechanism of wind-wave generation. The cited studies use a mix of wind-generated waves, mechanical waves, and numerically imposed Airy waves, and include wind speeds up to $U_{10}=26.9 \mathrm{~m} \mathrm{~s}^{-1}$ (Savelyev 2009; Savelyev et al. 2011).

\section{Error analysis}

In this section we test each of the major assumptions used in computing the pressure fields. We also use this to arrive at an approximate estimate of the errors involved in the pressure reconstruction. To do this we rely on comparisons with highly resolved large eddy simulation (LES) data of airflow over a fixed sinusoidal surface. The turbulent velocity and pressure fields produced in this way are expected to approximate that found at a fully coupled air-water interface (see Yang and Shen 2010; Liu et al. 2010; Sullivan et al. 2000). Parameters and boundary conditions in the LES are chosen to closely mimic conditions in the low wind speed wind-wave tank experiment. The mean velocity in the LES, when averaged over a region of 8.0 $9.7 \mathrm{~cm}$ above the solid boundary, is $1.45 \mathrm{~m} \mathrm{~s}^{-1}$, close to the $1.33 \mathrm{~m} \mathrm{~s}^{-1}$ found in the laboratory experiment. The wave slope in the LES of $a k=0.08$ also compares well to laboratory conditions of $a_{p} k_{p}=0.07$. See appendix B for details on the LES simulation.

When testing assumptions using the LES data, we input $x-z$ slices of the full, turbulent, three-dimensional LES flow into our Poisson solver and compare the resulting output with the exact pressure field output by the LES. In standard LES, the subgrid-scale (SGS) stress terms contribute to the forcing function $f$ of the pressure field, and can produce a so-called "modified pressure" by contributing to normal stresses. However, as described in appendix B, the resolution of our simulation is high 
enough that these SGS terms can be neglected except within a region very close to the bottom boundary. Therefore, in the vast majority of the domain outside the near-bottom boundary, we may refer to a pressure field (that does not include SGS terms) without ambiguity. Form drags from the LES are computed through eliminating the closest 5 grid points to the surface topography in $f$ in order to avoid numerical artifacts and SGS stresses close to the bottom boundary. We also evaluate the pressure gradients at this location to use as input to the solver as a bottom boundary condition. Form drag is then computed using the resulting pressure fields evaluated at the water/topography surface.

\section{a. Top boundary condition}

Figures $6 \mathrm{a}$ and $6 \mathrm{~b}$ show that, as expected, the choice of the top boundary influences the pressure fields near the top of the PIV domain. Near the water surface, however, the amplitude of the pressure field variations are comparable. When running these trials, $x-z$ slices are used that have the same height above the water surface as the smallest of the PIV fields. We therefore conclude that the top boundary condition does not affect the overall structure of the estimated pressure fields near the interface, provided that the domain extends sufficiently far above the interface. Note that it is the structure and variability of the surface pressure field that is most important for the momentum flux. The mean pressure has no influence on momentum or energy transfers across the air-water interface. It can be that errors in surface pressure are amplified by the surface slope which may lead to larger errors in the estimates of the form drag. In the LES simulations presented here, a $-25 \%$ error in the surface pressure amplitude yields a $-26 \%$ error in the estimated form drag.

This error can be reduced by placing the top boundary further from the water surface; however, we found a trade-off between error due to the side boundary condition and the top boundary condition as the height of the domain increases. In larger vertical domains, the error due to the top boundary decreases while the error in the side boundary approximation increases. Optimization of these errors could be performed in future studies, but was not done here.

\section{b. Side boundary condition}

Figure $6 \mathrm{c}$ shows how the approximate side boundary conditions further impact the pressure solution from the LES. Setting the side boundary conditions to $\nabla p \cdot \mathbf{n}=0$ introduces an additional error of only $+2 \%$ into the estimates of form drag for the LES pressure field. The additional error in the surface pressure is localized near the sides of the computational domain, and is largely neglected when only the central $60 \%$ of the domain is selected for further analysis.

\section{c. Bottom boundary condition}

The bottom boundary condition (i.e., on the water surface) relies on computing both the divergence of the surface viscous stress, and the acceleration of the interface [see Eq. (6)]. We discuss each of these in turn.

The sensitivity of the derived form drag to the viscous term in the bottom boundary condition was tested by applying various (artificial) amplifications of the term, corresponding to

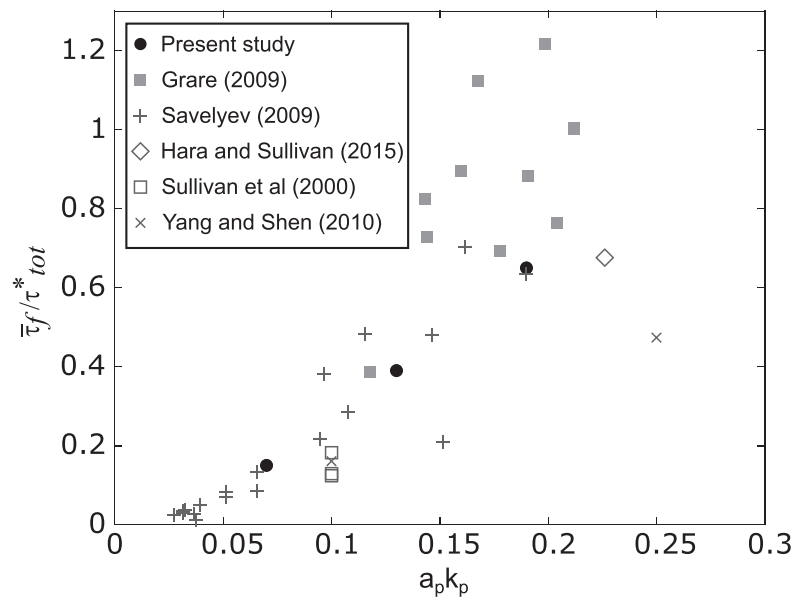

FIG. 5. Mean normalized form drag estimated using 2D pressure fields retrieved from $2 \mathrm{D}$ airflow PIV measurements over laboratory wind waves, normalized by the total stress, plotted vs wave slope (black symbols). Results from pressure measurements above mechanically generated waves from previous laboratory studies (Grare 2009; Savelyev 2009) are also shown, as well as previous form drag estimates from LES (Hara and Sullivan 2015) and DNS (Sullivan et al. 2000; Yang and Shen 2010).

two- and fivefold amplifications, as well as ignoring the term completely. The results of this analysis are shown in Table 3 , and demonstrate that changes in the mean form drag in each wind speed case are only larger than $4 \%$ of $\tau_{\text {tot }}^{*}$ for the fivefold amplification of the viscous term. We conclude that our results are not overly sensitive to errors in the computation of the viscous term, with errors of only a couple percent of total drag.

The acceleration of the airflow above the interface is due to both the orbital velocity from the propagating surface waves and to the acceleration and deceleration of the airflow (for these young waves) over the windward and leeward face of the waves, respectively. In the absence of wind, the pressure fluctuation at the air side of the water surface has an amplitude of $a g \rho$, with $a$ the wave amplitude, and $g$ the acceleration due to gravity (Kundu and Cohen 2002). Using values from Table 1 results in wave-induced pressure amplitudes of $0.018,0.060$, and $0.14 \mathrm{~Pa}$, for wind speeds of $U_{10}=2.2,5.0$, and $9.4 \mathrm{~m} \mathrm{~s}^{-1}$. These values are $37 \%, 6 \%$, and $3 \%$ of the amplitudes in the phase average pressure fields shown in Fig. 4, respectively. Thus, in all but the $U_{10}=2.2 \mathrm{~m} \mathrm{~s}^{-1}$ experiment, the movement of the surface is expected to have a minimal effect on the pressure field. In addition, sensitivity tests similar to those performed for the viscous term demonstrate that form drag estimates are not sensitive to an amplification of this term.

\section{d. The planar-f approximation}

Finally, we test the accuracy in reconstructing the pressure field using only the planar $(x, z)$ terms in the forcing function $f$ as in (4). This is done using the three-dimensional LES by comparing the form drags obtained directly from the simulated pressure field to those resulting from applying our 

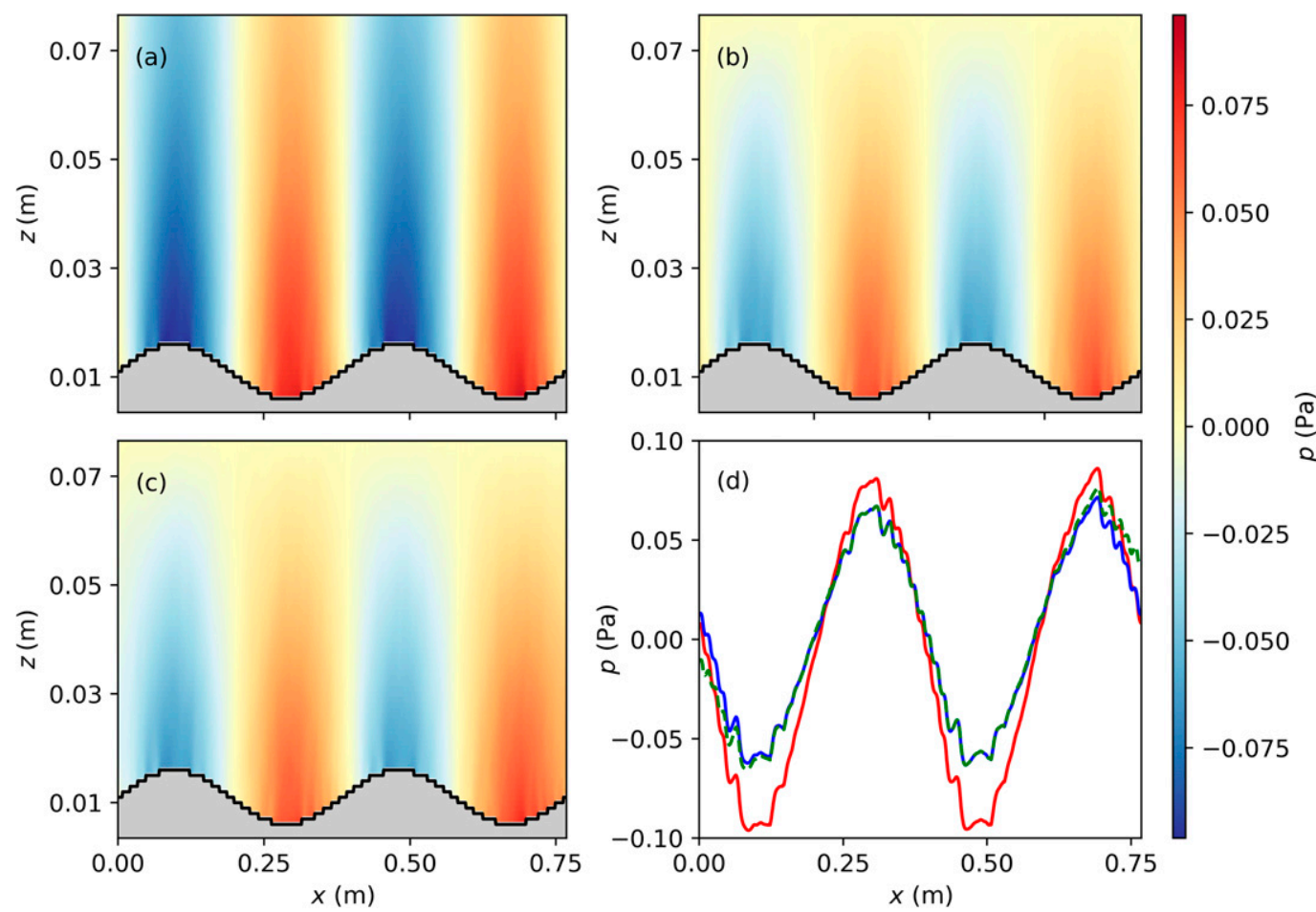

FIG. 6. Effect of boundary conditions on the pressure solution. (a) Time average pressure field obtained directly from the LES simulation; (b) pressure field obtained by using the flow output by the LES simulation in the Poisson solver, with the top boundary condition, $p=0$; (c) pressure field obtained by using the flow output by the LES simulation in the Poisson solver, with the top boundary condition $p=0$ and the side boundary condition $\nabla p \cdot \mathbf{n}=0$; (d) pressure variation along the water surface for each case: (a) red curve, (b) blue, (c) dashed green.

Poisson solver to planar $x-z$ slices. We have taken 450 total slices obtained from 150 different time steps over a 15 -s time window. The distributions of form drag obtained from these samples are shown in Fig. 7. It can be seen that the location of the means of the distributions are close to one another, differing by $-5 \%$. However, the much greater spread of the $\tau_{f}$ obtained from making the planar- $f$ approximation demonstrates that significant errors are present for instantaneous pressure fields, with rms relative errors for an instantaneous estimate of close to $300 \%$. Furthermore, it can be seen by the much smaller spread in the exact $\tau_{f}$ distribution (directly from the LES) that this is not due to a natural turbulent variability, but results from making the planar- $f$ approximation. Averaging over many fields is therefore required to produce an accurate estimate of form drag, and even with the 450 fields analyzed herein, the $95 \%$ bootstrapped confidence intervals give relative errors between $-33 \%$ and $22 \%$. This planar- $f$ approximation is therefore a significant source of error that requires much averaging over many hundreds of fields. This conclusion is in broad agreement with previous studies that have assessed the planar approximation in reproducing instantaneous pressure fluctuations (Van der Kindere et al. 2019). Note also that we have tested this approximation only for a single wind speed and wave slope, there could be a dependence on these variables, particularly when airflow separation is present. Analysis of these cases is, however, difficult due to the increased resolution needed to eliminate subgrid-scale effects of $f$.

\section{e. Summary of error estimates}

The major sources of error in reconstructed pressure fields were identified to be due largely to two effects: the top boundary condition, and the planar- $f$ approximation. The estimated absolute errors in mean form drag associated with all sources except the uncertain bottom boundary condition, amount to roughly $31 \%$. When converting these errors to percentages of the total drag $\tau_{\text {tot }}^{*}$, using the values of $\bar{\tau}_{f} / \tau_{\text {tot }}^{*}$ from Table 2 , and taking a $4 \%$ error for the bottom boundary

TABLE 3. Table of changes in the form drag $\bar{\tau}_{f}$, expressed as a percent of $\tau_{\text {tot }}^{*}$, obtained with various artificial amplifications of the viscous term in the bottom boundary condition. A $1 \times$ denotes the boundary condition as used throughout this analysis, and computed as described in section 3 , whereas $0 \times$ indicates the viscous term is set to zero.

\begin{tabular}{ccccc}
\hline \hline & \multicolumn{4}{c}{ Boundary amplification } \\
\cline { 2 - 5 }$U_{10}\left(\mathrm{~m} \mathrm{~s}^{-1}\right)$ & $0 \times$ & $1 \times$ & $2 \times$ & $5 \times$ \\
\hline 2.2 & $+2 \%$ & $0 \%$ & $-3 \%$ & $-9 \%$ \\
5.0 & $+4 \%$ & $0 \%$ & $-4 \%$ & $-16 \%$ \\
9.4 & $+4 \%$ & $0 \%$ & $-3 \%$ & $-11 \%$ \\
\hline
\end{tabular}




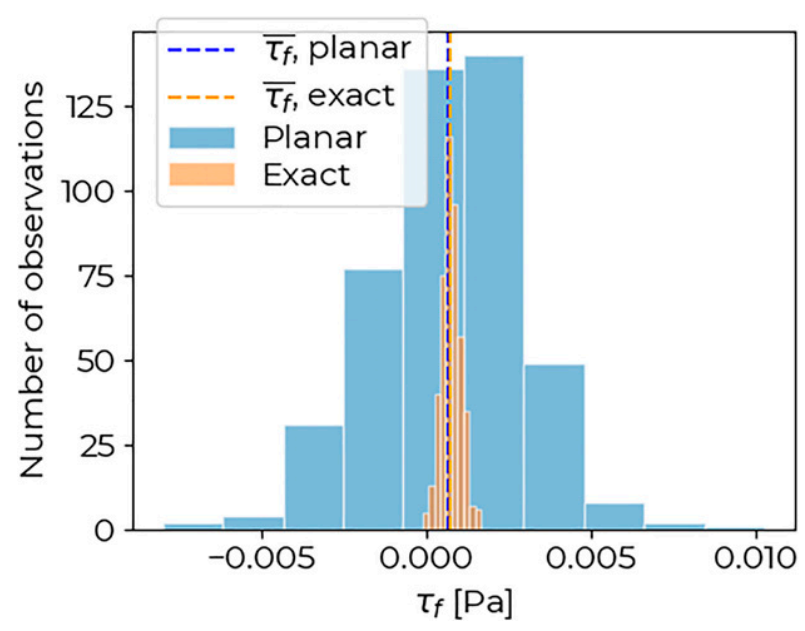

FIG. 7. Testing of the planar- $f$ approximation using threedimensional LES of turbulent flow over stationary sinusoidal topography. The two histograms indicate the distributions of form drag $\tau_{f}$, obtained directly from the LES pressure field (orange), and those obtained from using the planar- $f$ approximation. Means of each distribution are indicated by the dashed lines, and the sample size consists of $450 x-z$ slices. The means are located at $\bar{\tau}_{f}=7.5,7.1 \times 10^{-4} \mathrm{~Pa}$, for the exact LES and planar- $f$ approximation, respectively, and differ by $5 \%$.

condition, we arrive at an absolute error in total drag of $9 \%-$ $24 \%$. This is greater than the values reported in the final column of Table 2 , and suggests that the errors could be somewhat compensating. The largest unknown error source is likely to be the planar- $f$ approximation, which has large confidence intervals, and no definite sign. Note that this discussion above applies only to mean pressure fields. Errors for instantaneous fields are very large (i.e., hundreds of percent), and much averaging is required to produce accurate means.

\section{Summary and conclusions}

In this paper, we present a technique for reconstructing pressure fields in the airflow over water waves from combined PIV and LIF laboratory data. This is done through solving a pressure Poisson equation with a forcing that is determined through the relative strength of strain and vorticity (enstrophy) in the airflow. The method relies on the application of various approximate boundary conditions. Using the high resolution of the PIV measurements, and the pressure field estimates, it is possible to independently evaluate both the surface viscous stress and the pressure-induced form drag, thus estimating the total momentum flux at the interface. The results show that pressure-induced form drag becomes the greater momentum flux term at higher wind speeds (for $2.2 \leq U_{10} \leq 9.1 \mathrm{~m} \mathrm{~s}^{-1}$ ) and steeper wave slopes (for $0.07 \leq a_{p} k_{p} \leq 0.19$ ).

The agreement between the total stress, derived from the pressure reconstruction, with an independent law-of-the-wall bulk estimate, is within $5 \%$. This suggests that the pressure reconstruction technique is able to capture the wave-phase coherent variations of the pressure field at the interface. However, the analysis of the different approximations used in the reconstruction show that the two main sources of error, which result from the top boundary condition and the planar approximation, have larger magnitudes (with the sum of the errors at $31 \%$ of total form drag), and may partially compensate. It is not clear that these errors would still compensate in different wind sea conditions (older wave ages for example), and the errors in the pressure reconstruction may increase. Particularly uncertain is the planar approximation, which could exhibit bias due to out-of-plane components when wind and waves are not aligned. We note, however, that larger PIV domains that reach heights beyond the wave boundary layer will reduce the errors associated with the top boundary condition.

The ability to directly estimate pressure fields in the vicinity of the air-water interface using PIV-LIF measurements opens up new possibilities for studying the mechanisms of momentum and energy transfers between the atmosphere and the ocean. Although such mechanisms have long been the subject of numerous studies, direct measurements of the pressure field have been lacking. This work therefore fills an important gap in the physics of atmosphere-ocean coupling.

Acknowledgments. We would like to acknowledge financial support from the Helmholtz Association through the PACES II programme, as well as the German Research Foundation (DFG). This paper is a contribution to the project M6 and T4 of the Collaborative Research Centre TRR 181, "Energy Transfers in Atmosphere and Ocean" funded through DFG Grant 274762653. MPB and FV acknowledge support from the U.S. National Science Foundation (Grants OCE-1458977, OCE-1233808, OCE-0748767, and AGS-PRF-1524733).

Data availability statement. The PIV-LIF data fields used in this study are too large to be stored in a public repository; data can be made available upon request to Marc P. Buckley (marc. buckley@hereon.de).The input files required to reproduce the LES can be found on the Zenodo repository at the DOI 10.5281/zenodo.5355659.

\section{APPENDIX A}

\section{Derivation of Momentum Fluxes}

We denote the two dimensional stress tensor for incompressible flows by

$$
\boldsymbol{\tau}_{i j}=\left[\begin{array}{cc}
-p+2 \mu \frac{\partial u}{\partial x} & \mu\left(\frac{\partial u}{\partial z}+\frac{\partial w}{\partial x}\right) \\
\mu\left(\frac{\partial u}{\partial z}+\frac{\partial w}{\partial x}\right) & -p+2 \mu \frac{\partial w}{\partial z}
\end{array}\right],
$$

with $\mu$ the dynamic viscosity of the fluid (air, in the present case). The total flux of horizontal momentum through the water surface can then be calculated through

$$
\boldsymbol{\tau}_{\mathrm{tot}}=\int \boldsymbol{\tau}_{1 j} \cdot \mathbf{n} d s
$$

using the sign convention that positive $\tau$ corresponds to a horizontal momentum loss by the air, and gain by the water. 
Here we define $\mathbf{n}$ as the upward pointing unit normal to the water surface, represented by $s$. In practice, we evaluate this integral using instantaneous measurements of the water surface elevation, $\eta(x, t)$, by substituting

$$
\mathbf{n}=\left(-\frac{\partial \eta}{\partial x}, 1\right) /\left[\left(\frac{\partial \eta}{\partial x}\right)^{2}+1\right]^{1 / 2}
$$

and using the relation

$$
\int f[x, \eta(x, t)] d s=\int f[x, \eta(x, t)]\left[\left(\frac{\partial \eta}{\partial x}\right)^{2}+1\right]^{1 / 2} d x .
$$

This leads to the following form for total horizontal stress

$$
\tau_{\text {tot }}=\underbrace{\int \mu\left(\frac{\partial u}{\partial z}+\frac{\partial w}{\partial x}-2 \frac{\partial u}{\partial x} \frac{\partial \eta}{\partial x}\right) d x}_{\text {viscous stress }}+\underbrace{\int p \frac{\partial \eta}{\partial x} d x}_{\text {form drag }}
$$

being composed of both pressure (form drag) and viscous components, as indicated.

\section{APPENDIX B}

\section{Description of the LES}

The large-eddy simulation (LES) method is based on the concept of the energy cascade, in which flow instabilities break down large eddies into smaller eddies and so transfer their energy to smaller scales. In LES, the contribution of the small eddies is parameterized through a subgrid-scale (SGS) model, whereas the large eddies are resolved. We use the Parallelized Large-Eddy Simulation Model for atmospheric and oceanic flows (PALM, version 6.0, revision 4901), developed at the Institute of Meteorology and Climatology of the Leibniz University of Hannover (Raasch and Schröter 2001; Maronga et al. 2015).

The domain described for the simulations had dimensions of $0.768 \mathrm{~m} \times 0.128 \mathrm{~m} \times 0.256 \mathrm{~m}$ along $x, y$, and $z$, respectively. The grid size was set to $1 \mathrm{~mm}$ in all directions. Two sinusoidal waves of length $38.4 \mathrm{~cm}$ and crest-to-trough height of $1.0 \mathrm{~cm}$ were prescribed along the mean flow $x$ direction, giving a wave slope of $a k=0.082$. A horizontal mean pressure gradient was applied to allow the mean flow to achieve a quasi-steady state.

The lateral boundaries of the domain were assigned periodic conditions, with a velocity bottom boundary condition prescribed by Monin-Obukhov similarity using a nondimensional roughness length of $k z_{0}=1.1 \times 10^{-4}$, which prescribes a relationship between the surface momentum flux and the tangential velocity at the first grid level. The roughness length was chosen to match the value of the low wind speed experiment (see Buckley et al. 2020). At the domain top, a stress-free, rigid lid was used for the velocity. To initiate the development of turbulence, random perturbations in the velocity field with a magnitude of up to $0.02 \mathrm{~m} \mathrm{~s}^{-1}$ were imposed at the initial time. A minimum level of subgrid-scale turbulent kinetic energy (SGS-TKE) was set in order to limit the eddy viscosity to its molecular value. This state of minimum SGS-TKE was found throughout the majority of the domain during the quasi-steady period in which the analysis was performed. The simulations are, in this respect, more representative of direct numerical simulations, where the smallest turbulent eddies are resolved, and there is no need for subgrid-scale momentum fluxes. This also ensures that there is minimal influence of the SGS terms in the pressure reconstruction.

\section{REFERENCES}

Ardhuin, F., B. Chapron, and T. Elfouhaily, 2004: Waves and the air-sea momentum budget: Implications for ocean circulation modeling. J. Phys. Oceanogr., 34, 1741-1755, https://doi.org/ 10.1175/1520-0485(2004)034<1741:WATAMB >2.0.CO;2.

Banner, M. L., and W. L. Peirson, 1998: Tangential stress beneath wind-driven air-water interfaces. J. Fluid Mech., 364, 115-145, https://doi.org/10.1017/S0022112098001128.

Buckley, M. P., and F. Veron, 2016: Structure of the airflow above surface waves. J. Phys. Oceanogr., 46, 1377-1397, https:// doi.org/10.1175/JPO-D-15-0135.1.

$\longrightarrow$, and —, 2017: Airflow measurements at a wavy air-water interface using PIV and LIF. Exp. Fluids, 58, 161, https:// doi.org/10.1007/s00348-017-2439-2.

— surface waves. Eur. J. Mech., 73B, 132-143, https://doi.org/ 10.1016/j.euromechflu.2018.04.003.

,-- , and K. Yousefi, 2020: Surface viscous stress over winddriven waves with intermittent airflow separation. J. Fluid Mech., 905, A31, https://doi.org/10.1017/jfm.2020.760.

Cavaleri, L., B. Fox-Kemper, and M. Hemer, 2012: Wind waves in the coupled climate system. Bull. Amer. Meteor. Soc., 93, 1651-1661, https://doi.org/10.1175/BAMS-D-11-00170.1.

Davidson, P., 2015: Turbulence: An Introduction for Scientists and Engineers. Oxford University Press, 630 pp.

Dobson, F. W., 1971: Measurements of atmospheric pressure on wind-generated sea waves. J. Fluid Mech., 48, 91-127, https:// doi.org/10.1017/S0022112071001496.

Donelan, M. A., 1998: Air-water exchange processes. Physical Processes in Lakes and Oceans, J. Imberger, Ed., Coastal and Estuarine Studies, Vol. 54, Amer. Geophys. Union, 19-36.

_ A. V. Babanin, I. R. Young, and M. L. Banner, 2006: Wavefollower field measurements of the wind-input spectral function. Part II: Parameterization of the wind input. J. Phys. Oceanogr., 36, 1672-1689, https://doi.org/10.1175/JPO2933.1.

Elliott, J., 1972: Microscale pressure fluctuations near waves being generated by the wind. J. Fluid Mech., 54, 427-448, https:// doi.org/10.1017/S0022112072000783.

Fox, L., 1944: Solution by relaxation methods of plane potential problems with mixed boundary conditions. Quart. Appl. Math., 2, 251-257, https://doi.org/10.1090/qam/10679.

Grare, L., 2009: Etude des interactions océan-atmosphère à proximité immédiate de l'interface: Application aux vagues de vent et aux vagues extrêmes. Ph.D. thesis, University of AixMarseille, 222 pp.

—, W. L. Peirson, H. Branger, J. W. Walker, J.-P. Giovanangeli, and V. Makin, 2013: Growth and dissipation of wind-forced, deep-water waves. J. Fluid Mech., 722, 5-50, https://doi.org/ 10.1017/jfm.2013.88.

Hao, X., and L. Shen, 2019: Wind-wave coupling study using les of wind and phase-resolved simulation of nonlinear waves. J. Fluid Mech., 874, 391-425, https://doi.org/10.1017/jfm.2019.444.

Hara, T., and P. P. Sullivan, 2015: Wave boundary layer turbulence over surface waves in a strongly forced condition. J. Phys. Oceanogr., 45, 868-883, https://doi.org/10.1175/JPO-D-14-0116.1. 
Hasselmann, D., and J. Bösenberg, 1991: Field measurements of wave-induced pressure over wind-sea and swell. J. Fluid Mech., 230, 391-428, https://doi.org/10.1017/S0022112091000848.

Husain, N. T., T. Hara, M. P. Buckley, K. Yousefi, F. Veron, and P. P. Sullivan, 2019: Boundary layer turbulence over surface waves in a strongly forced condition: Les and observation. J. Phys. Oceanogr., 49, 1997-2015, https://doi.org/10.1175/JPO-D-19-0070.1.

Kitaigorodskii, S., and M. A. Donelan, 1984: Wind-wave effects on gas transfer. Gas Transfer at Water Surfaces, Springer, 147-170.

Kundu, P. K., and I. M. Cohen, 2002: Fluid Mechanics. Academic Press, $872 \mathrm{pp}$.

Liu, Y., D. Yang, X. Guo, and L. Shen, 2010: Numerical study of pressure forcing of wind on dynamically evolving water waves. Phys. Fluids, 22, 041704, https://doi.org/10.1063/1.3414832.

Maronga, B., and Coauthors, 2015: The Parallelized Large-Eddy Simulation Model (PALM) version 4.0 for atmospheric and oceanic flows: Model formulation, recent developments, and future perspectives. Geosci. Model Dev., 8, 2515-2551, https:// doi.org/10.5194/gmd-8-2515-2015.

Morton, K. W., and D. F. Mayers, 2005: Numerical Solution of Partial Differential Equations. Cambridge University Press, 278 pp.

Murai, Y., T. Nakada, T. Suzuki, and F. Yamamoto, 2007: Particle tracking velocimetry applied to estimate the pressure field around a Savonius turbine. Meas. Sci. Technol., 18, 2491-2503, https://doi.org/10.1088/0957-0233/18/8/026.

Noye, B., and R. Arnold, 1990: Accurate finite difference approximations for the Neumann condition on a curved boundary. Appl. Math. Model., 14, 2-13, https://doi.org/10.1016/0307904X(90)90157-Z.

Phillips, O. M., 1977: The Dynamics of the Upper Ocean. Cambridge University Press, $261 \mathrm{pp}$.

Raasch, S., and M. Schröter, 2001: PALM - A large eddy simulation model performing on massively parallel computers. Meteor. Z., 10, 363-372, https://doi.org/10.1127/0941-2948/ 2001/0010-0363.

Savelyev, I. B., 2009: A laboratory study of the transfer of momentum across the air-sea interface in strong winds. Ph.D. thesis,
University of Miami, 101 pp., https://scholarship.miami.edu/ esploro/outputs/doctoral/A-Laboratory-Study-of-the-Transfer/ 991031447464502976.

— B. K. Haus, and M. A. Donelan, 2011: Experimental study on wind-wave momentum flux in strongly forced conditions. J. Phys. Oceanogr., 41, 1328-1344, https://doi.org/10.1175/ 2011JPO4577.1.

Sullivan, P. P., and J. McWilliams, 2010: Dynamics of winds and currents coupled to surface waves. Annu. Rev. Fluid Mech., 42, 19-42, https://doi.org/10.1146/annurev-fluid-121108-145541.

— J. J. McWilliams, and C.-H. Moeng, 2000: Simulation of turbulent flow over idealized water waves. J. Fluid Mech., 404, 47-85, https://doi.org/10.1017/S0022112099006965.

_ M. L. Banner, R. P. Morison, and W. L. Peirson, 2018: Turbulent flow over steep steady and unsteady waves under strong wind forcing. J. Phys. Oceanogr., 48, 3-27, https:// doi.org/10.1175/JPO-D-17-0118.1.

Van der Kindere, J. W., A. Laskari, B. Ganapathisubramani, and R. de Kat, 2019: Pressure from 2D snapshot PIV. Exp. Fluids, 60, 32, https://doi.org/10.1007/s00348-019-2678-5.

Van Oudheusden, B., 2013: PIV-based pressure measurement. Meas. Sci. Technol., 24, 032001, https://doi.org/10.1088/09570233/24/3/032001.

Veron, F., G. Saxena, and S. K. Misra, 2007: Measurements of the viscous tangential stress in the airflow above wind waves. Geophys. Res. Lett., 34, L19603, https://doi.org/10.1029/2007GL031242.

Wunsch, C., and R. Ferrari, 2004: Vertical mixing, energy, and the general circulation of the oceans. Annu. Rev. Fluid Mech., 36, 281-314, https://doi.org/10.1146/annurev.fluid.36.050802.122121.

Yang, D., and L. Shen, 2010: Direct-simulation-based study of turbulent flow over various waving boundaries. J. Fluid Mech., 650, 131-180, https://doi.org/10.1017/S0022112009993557.

Young, W., and C. Wolfe, 2014: Generation of surface waves by shear-flow instability. J. Fluid Mech., 739, 276-307, https:// doi.org/10.1017/jfm.2013.617.

Zhang, W., W. Perrie, and W. Li, 2006: Impact of waves and sea spray on midlatitude storm structure and intensity. Mon. Wea. Rev., 134, 2418-2442, https://doi.org/10.1175/MWR3191.1. 